\title{
Analysis of Renewable Energy in Four States of USA
}

\author{
Bingjun Li, Yuan Wang, Yiteng Gao and Linke Zhu \\ Department of Mathematics, Hunan Institute of Humanities, Science and Technology, Loudi City, Hunan, China 417000
}

\begin{abstract}
Four states, California (CA), Arizona (AZ), new Mexico (NM) and Texas (Texas), have to use clean and renewable energy to form a new energy contract for the purpose of solving the energy challenge. Due to the complexity of the influencing factors, regression models may lead to deviations, so factor analysis is used to calculate the contribution of each factor. We conclude that the following 5 factors: TEICB (total energy consumed in industrial sector), TERCB (residential sector of total energy consumption), TEEIB (total energy of electric power consumption), AR (average rainfall), AWS (average wind speed) affect the similarities and differences of the four states at most.
\end{abstract}

Keywords-entropy weight coefficient method model; regression model; clean and renewable energy

\section{INTRODUCTION}

Energy is an important material basis for people's survival. It is also the basis for all countries to achieve economic growth and promote social development. The sustainable development of a country cannot do without sufficient energy security. With the rapid development of modern society, the human demand for energy is increasing day by day. However, the contradiction between the limited traditional energy and the energy needed for its development has become increasingly prominent. As a result, green energy such as clean energy and renewable resources has become the focus of international attention today. More countries will save energy, develop renewable resources and clean energy as a long-term strategy for energy development.

The demand for energy of a country will directly affect the formulation and implementation of energy policy such as energy strategy planning, energy supply and demand distribution and energy conservation and emission reduction. To do a good job in the above planning is of great significance to the sustained and healthy economic development of a country. Obviously, different regions and industries in different countries also affect energy use and production. On the border between the United States and Mexico, there are four states California (CA), Arizona (AZ), New Mexico (NM), and Texas. This paper discusses the widespread use of clean and renewable energy form a realistic new energy contract.

In this paper we determine which factors affect the similarities and differences of the four states at most.

\section{MAIN RESULTS}

We compare the energy use in each state, including the total consumption of different types of energy and total expenditure. Similarities and differences can be found between the four states. Among them, state renewable energy is gradually developing, but the development trend is different. We want to find out the reasons for the differences, we can use one-way analysis of variance, under the influence of some factors, come to the corresponding results. After summarizing, we can provide the Governor with a variety of reasonable suggestions.

\section{A. Unidirectional Analysis of Variance}

One-way ANOVA focused on the comparison of two or more groups. Let

$$
I=\text { the number of treatments being compared }
$$

$$
J=\text { the number of observations in each sample }
$$

$\mu_{1}=$ the mean of population 1 (or the true average response when treatm 1 is applied)

$\mu_{4}=$ the mean of population 4(or the true average response when treatment 4 is applied)

So the assumption of interest is

$$
H_{0}: \mu_{1}=\mu_{2}=\mu_{3}=\mu_{4}
$$

versus

$$
H_{a}: \text { at least two of the } \mu_{i} \text { 's are different }
$$

Testing these hypotheses requires that we randomly select samples from each population or treatment.

In the double-sample problem, we use the letters $\mathrm{X}$ and $\mathrm{Y}$ to specify the observations in both samples. Since this is cumbersome for three or more samples, it is customary to use a single letter with two subscripts. The first subscript identifies the sample number, corresponding to the population or process being sampled, and the second subscript indicates the position observed within the sample. Let 


$$
\begin{aligned}
& \left.X_{i j}=\text { the random variable ( } r v\right) \text { denoting } \\
& \text { the jth measurement form the ith population }
\end{aligned}
$$

$$
\begin{aligned}
& X_{i j}=\text { the observed value of } \\
& X_{i j} \text { when the experim ent is performed }
\end{aligned}
$$

In some $\overline{X_{1}}, \overline{X_{2}}, \overline{X_{3}}, \overline{X_{4}}$, experiments, different samples contained different numbers of observations. However, concepts and methods of ANOVA are most easily developed for the same sample size. Limit us temporarily to the sample size, let $\mathrm{J}$ denote the number of observations in each sample. The dataset consists of IJ observations. Means of personal sample Will be represented as, ie,

$$
\overline{X_{i \bullet}}=\frac{\sum_{j=1}^{J} X_{i j}}{J} \quad i=1,2,3,4
$$

The point in place of the second subscript indicates that we have added subscripts to all subscript values While other subscript value is fixed, the horizontal bar representation divided by J gives an average. Similarly, the average of all IJ observations, called the large mean, is

$$
\overline{X_{. .}}=\frac{\sum_{i=1}^{I} \sum_{j=1}^{J} X_{i j}}{I J}
$$

Squared processing and SSTr are given by:

$$
\operatorname{SSTr}=J \sum\left(\overline{X_{m}}-\bar{X}_{m, m}\right)^{2}=J\left[\left(\bar{X}_{1 n}-\bar{X}_{m, m}\right)^{2}+\cdots+\left(\overline{X_{4 n}}-\bar{X}_{m, m}\right)^{2}\right]
$$

And the square error sum SSE is

$$
\begin{aligned}
S S E & =\sum_{i} \sum_{j}\left(X_{i j}-\overline{X_{i}}\right)^{2} \\
& =\sum_{j}\left(X_{1 j}-\overline{X_{1}}\right)^{2}+\cdots+\sum_{j}\left(X_{4 j}-\overline{X_{4}}\right)^{2} \\
& =(J-1) S_{1}^{2}+(J-1) S_{2}^{2}+\cdots+(J-1) S_{4}^{2} \\
& =(J-1)\left[S_{1}^{2}+S_{2}^{2}+S_{3}^{2}+S_{4}^{2}\right]
\end{aligned}
$$

The formula for calculating SSE is the result of basic ANOVA identification.

$$
S S T=S S T r+S S E
$$

The mean square error of treatment $\operatorname{MSTR}=\operatorname{SSTr} /(I-1)$ is the mean square $M S E=S S /[I(J-1)]$ error

The test statistic for the one-way ANOVA was. The Pvalue of the upper tail $F$ test was calculated $f$ for the area under the relevant F-curve (below the correct value for numerator and denominator $\mathrm{df}$. We use the $\mathrm{P}$ value to measure the result.

\section{B. Results}

Here we use TEICB as an example to show the results of one-way ANOVA.

The $\mathrm{P}$ values for all factors are as follows.

When $\mathrm{P}<0.05$, there is a significant difference between these four states.

- TEICB (total energy consumed in the industrial sector).

- TERCB (Total energy consumed by the residential sector).

- TEEIB (total energy consumed by the power sector).

- AR (average rainfall)

- AWS (average wind speed)

\section{REFERENCES}

[1] https://www.eia.gov/state/seds/sep_use/notes/use_tot.pdf

[2] http://westernenergyboard.org/wieb-board/who-what/

[3] https://en.wikipedia.org/wiki/Geography_of_Arizona

[4] https://en.wikipedia.org/wiki/Geography_of_California

[5] https://en.wikipedia.org/wiki/Climate_of_Texas

[6] https://wrcc.dri.edu/narratives/NEWMEXICO.htm

[7] Yi Desheng Guo Ping: Grey Theory and Method, Petroleum Industry Press, 1992.

[8] MATLAB Chinese Forum: Thirty Cases Analysis of MATLAB Neural Network, Beijing University of Aeronautics and Astronautics Press, 2010. 